\title{
Electrothermal Feedback and Absorption-Induced Open-Circuit-Voltage Turnover in Solar Cells
}

\author{
Sascha Ullbrich, ${ }^{1, *}$ Axel Fischer, ${ }^{1, \dagger}$ Zheng Tang, ${ }^{1}$ Jorge Ávila, ${ }^{2}$ Henk J. Bolink, ${ }^{2}$ Sebastian Reineke, ${ }^{1}$ and Koen Vandewal ${ }^{1}$ \\ ${ }^{1}$ Dresden Integrated Center for Applied Physics and Photonic Materials (IAPP) \\ and Institute for Applied Physics, Technische Universität Dresden, \\ Nöthnitzer Straße 61, 01187 Dresden, Germany \\ ${ }^{2}$ Instituto de Ciencia Molecular (ICMol), Universidad de Valencia, Calle Catedrático José Beltrán 2 , \\ 46980 Paterna, Spain
}

(Received 25 July 2017; revised manuscript received 1 December 2017; published 31 May 2018)

\begin{abstract}
Solar panels easily heat up upon intense solar radiation due to excess energy dissipation of the absorbed photons or by nonradiative recombination of charge carriers. Still, photoinduced self-heating is often ignored when characterizing lab-sized samples. For light-intensity-dependent measurements of the opencircuit voltage (Suns- $V_{\mathrm{OC}}$ ), allowing us to characterize the recombination mechanism, sample heating is often not considered, although almost $100 \%$ of the absorbed energy is converted into heat. Here, we show that the frequently observed stagnation or even decrease in $V_{\mathrm{OC}}$ at increasingly high light intensities can be explained by considering an effective electrothermal feedback between the recombination current and the open-circuit voltage. Our analytical model fully explains the experimental data for various solar-cell technologies, comprising conventional inorganic semiconductors as well as organic and perovskite materials. Furthermore, the model can be exploited to determine the ideality factor, the effective gap, and the temperature rise from a single Suns- $V_{\mathrm{OC}}$ measurement at ambient conditions.
\end{abstract}

DOI: 10.1103/PhysRevApplied.9.051003

Photovoltaic devices convert absorbed photons from the Sun into electrical power. During this process, unavoidable thermalization processes lead to self-heating. For silicon solar cells, the relaxation of charge carriers created by photons with energies larger than the optical gap accounts for about $30 \%$ of the absorbed energy [1]. Furthermore, the radiative fraction is well below $1 \%$ of the total recombination current for most technologies [2]. This leaves nonradiative losses as the dominant recombination mechanism, leading to a temperature increase of photovoltaic devices under illumination. The influence of temperature on solar-cell parameters, for instance on the open-circuit voltage, is well known [3]. For most material systems, higher temperatures are unfavorable for the powerconversion efficiency due to the decrease in $V_{\mathrm{OC}}$, making active cooling of the devices an option [4,5]. In a few cases, some technologies, such as organic solar cells, can benefit

\footnotetext{
*Corresponding author. sascha.ullbrich@iapp.de

Corresponding author. axel.fischer@iapp.de

Published by the American Physical Society under the terms of the Creative Commons Attribution 4.0 International license. Further distribution of this work must maintain attribution to the author(s) and the published article's title, journal citation, and DOI.
}

from higher temperatures, at which thermally activated hopping transport becomes more efficient, resulting in increased fill factors, which can counteract the drop in $V_{\mathrm{OC}}$ in limited temperature ranges [6,7].

At open circuit, almost $100 \%$ of the energy is converted into heat since an external current flow is suppressed. As a consequence, internal or external series resistances do not alter the open-circuit voltage $\left(V_{\mathrm{OC}}\right)$, a fact exploited in Suns- $V_{\mathrm{OC}}$ measurements $[8,9]$. With this technique, the $V_{\mathrm{OC}}$ is recorded for various intensities and photocurrents, yielding a pseudo-current-voltage curve that follows the Shockley equation for open-circuit conditions, i.e., $J\left(V_{\mathrm{OC}}\right)=0$ :

$$
J_{\mathrm{ph}}=J_{00} \exp \left(\frac{q V_{\mathrm{OC}}-E_{a}}{n_{\mathrm{id}} k T}\right) .
$$

Hereby is $J_{\text {ph }}$ the photogenerated current at a specific incident illumination intensity $\left(I_{\text {Sun }}\right)$ and $E_{a}$ describes an activation energy corresponding to the effective band gap at which recombination takes place, while $J_{00}$ is the maximum theoretical recombination current. Suns- $V_{\mathrm{OC}}$ or the more elaborate $J_{\mathrm{sc}}-V_{\mathrm{OC}}$ measurement are widely used among many material systems and solar-cell communities. These techniques have been introduced to study recombination processes, e.g., by determining the ideality factor $n_{\text {id }}$, or to obtain intrinsic charge-carrier densities of organic solar cells $[10,11]$. Temperature-dependent Suns- $V_{\mathrm{OC}}$ measurements 


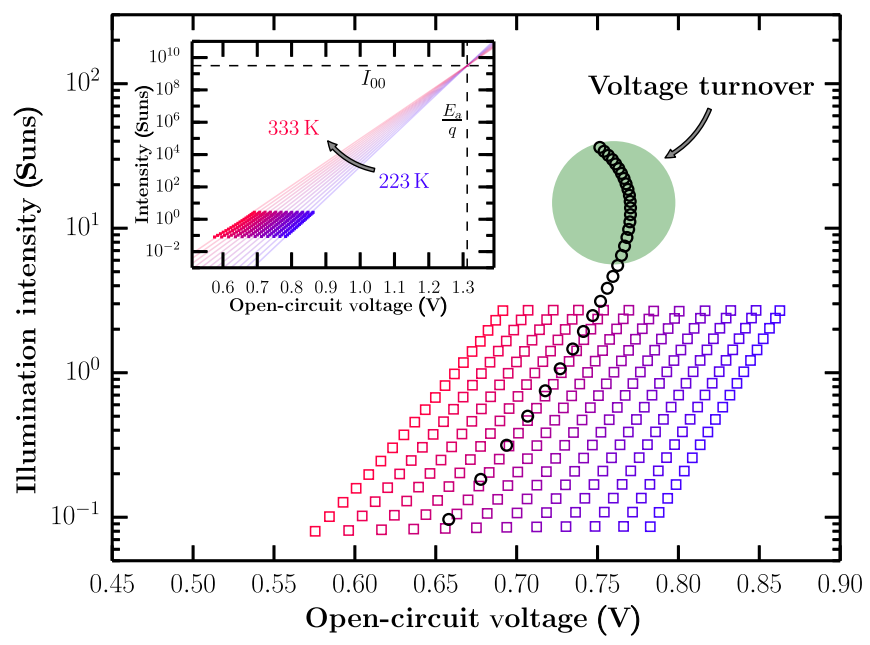

FIG. 1. Suns- $V_{\mathrm{OC}}$ measurement of an organic solar cell based on a $\mathrm{F} 4-\mathrm{ZnPc}: \mathrm{C}_{60}$ blend and an area of $6.44 \mathrm{~mm}^{2}$ for different ambient temperatures (colored squares) from 223 to $333 \mathrm{~K}$ in steps of $10 \mathrm{~K}$. Using a global fitting routine according to Eq. (1), it is possible to determine the activation energy $E_{a}$. Black circles present a Suns- $V_{\text {OC }}$ measurement taken at ambient temperatures using a brighter illumination source. At high light intensities, saturation of $V_{\mathrm{OC}}$ and a turnover is observed.

(see colored squares in Fig. 1) additionally allow us to determine $J_{00}$ and the effective gap $E_{a}$ of a solar cell [12].

Although Suns- $V_{\mathrm{OC}}$ measurements are valuable, the effect of sample heating has not been discussed thoroughly, despite using illumination intensities of up to 1000 Sun equivalents [13,14]. At high applied intensities, an absorption-induced self-heating of the device becomes likely, ultimately leading to a decreased $V_{\text {OC }}$. Indeed, a saturation of $V_{\mathrm{OC}}$ has frequently been observed at strong irradiation, but has been typically attributed to surface recombination rather than heating [15-17]. Moreover, voltage turnovers at increasing light intensities (see black circles in Fig. 1) have been observed for both inorganic $[13,14,18]$ and organic solar cells [12]. Again, this effect is assigned to the contacts and can be modeled as a Schottky diode and parallel shunt resistor equivalent circuit, reducing the voltage at high intensities [13], or it can be an intrinsic feature of silicon heterojunction solar cells [19].

In this work, we provide an alternative simple analytical model describing these voltage turnover effects in Suns- $V_{\mathrm{OC}}$ measurements (black circles in Fig. 1) and show that such a behavior can often be solely explained by an electrothermal feedback effect. We validate this model for various solar-cell technologies including smallmolecule organic solar cells, perovskite solar cells, and silicon solar cells. Furthermore, understanding the absorption-induced self-heating has also practical significance. The distinct shape of the curve allows us to determine the ideality factor, the relevant energy gap, and the temperature rise from a single measurement at ambient conditions. Thus, the presented adapted Suns- $V_{\mathrm{OC}}$ technique allows us to quickly compare different materials and structures regarding these basic parameters without requiring an external temperature control.

In order to demonstrate this method, we first perform a set of Suns- $V_{\mathrm{OC}}$ measurements where we continuously illuminate the device with a fixed intensity for a hold time of $30 \mathrm{~s}$ and subsequently record $V_{\mathrm{OC}}$. The light intensity is measured with a photodiode and calibrated to Sun equivalents based on mismatch-corrected $J(V)$ characteristics (see Supplemental Material, part III [20]). The red circles in Fig. 2 correspond to these continuous Suns- $V_{\mathrm{OC}}$ measurements, which reproduce the typical voltage saturation and turnover for various solar-cell technologies [more details and $J(V)$ characteristics can be found in the Supplemental Material, part I [20] ].

In order to explain this observation, we invoke selfheating effects and consider the following: A temperature change $\Delta T$ caused by absorption-induced self-heating is given by the product of the dissipating power and a thermal
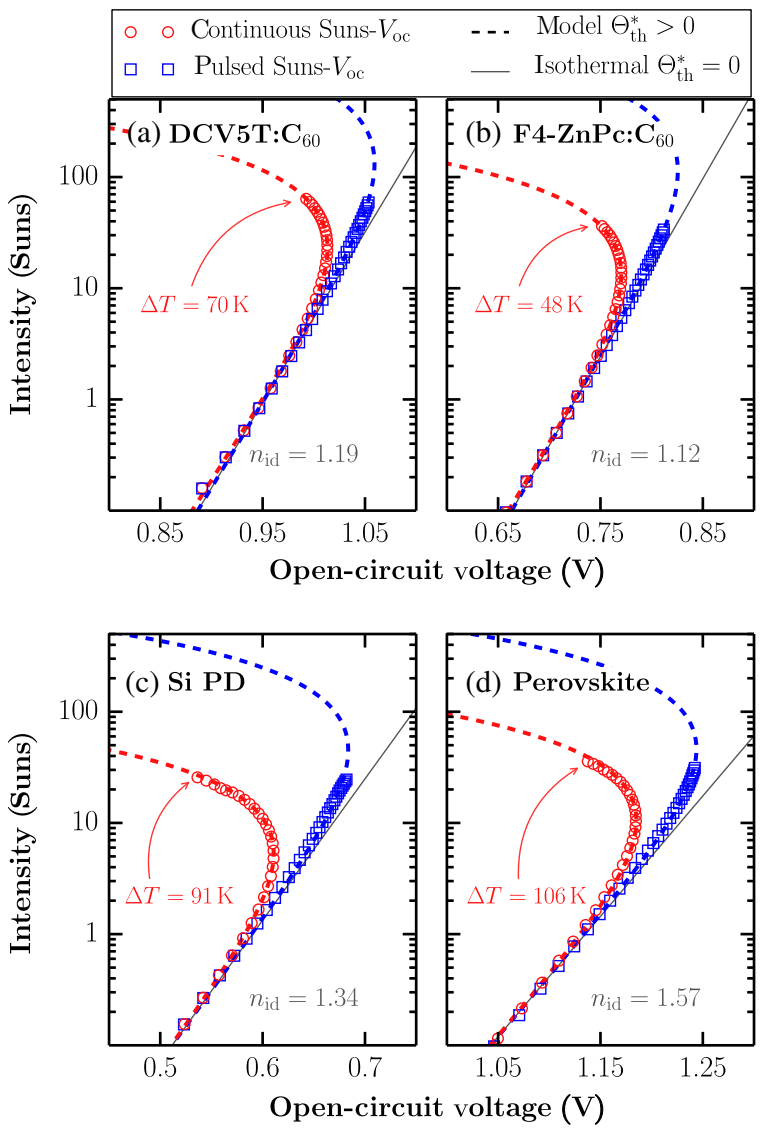

FIG. 2. Suns- $V_{\mathrm{OC}}$ measurements of (a) organic solar cell comprising a blend of DCV5T as donor and $\mathrm{C}_{60}$ as acceptor material; (b) organic solar cell with $\mathrm{F} 4-\mathrm{ZnPc}$ as donor and $\mathrm{C}_{60}$ as acceptor; (c) silicon photo diode; (d) perovskite solar cell. Red circles show a voltage turnover which can be explained by absorption-induced self-heating. For comparison, blue squares depict a measurement with a cooldown time between each data point with reduced electrothermal feedback. The latter lay closer to the isothermal curve. 
resistance $\Theta_{\text {th }}$. The thermal resistance depends on various parameters, such as the substrate geometry and the heat dissipation to the environment and can be time dependent $[21,22]$. By definition, there is no current flow at opencircuit conditions, which means that almost all absorbed energy is eventually converted into heat. It, therefore, can be described by the absorption $A$, the photon flux $\Phi$ of the light source, and the energy of the photons $E_{\mathrm{ph}}$ :

$$
\Delta T=\Theta_{\mathrm{th}} \int A(\lambda) \Phi(\lambda) E_{\mathrm{ph}}(\lambda) d \lambda=\underbrace{\Theta_{\mathrm{th}} \tilde{A}}_{\Theta_{\mathrm{th}}^{*}} I_{\text {Sun }} .
$$

Typically, solar cells are characterized under standard reporting conditions, i.e., the photon flux is well defined by the AM1.5 spectrum [23], while the product of photon flux and energy describes the intensity $I_{\text {Sun }}$ in units of Sun equivalents. For simplicity, it is convenient to introduce a reduction factor $\tilde{A}$ accounting for the fraction of incident energy that is absorbed, leading to an effective thermal resistance $\Theta_{\mathrm{th}}^{*}$ as fitting parameter. Inserting Eq. (2) into the Shockley equation [Eq. (1)], assuming that the photogenerated current is proportional to the incident illumination intensity, i.e., $J_{\mathrm{ph}} \propto I_{\mathrm{Sun}}$, and solving it for $V_{\mathrm{OC}}$ yields

$V_{\mathrm{OC}}\left(I_{\text {Sun }}\right)=\left(T_{\mathrm{amb}}+I_{\mathrm{Sun}} \Theta_{\mathrm{th}}^{*}\right) \frac{n_{i d} k}{q} \ln \left(\frac{I_{\mathrm{Sun}}}{I_{00}}\right)+\frac{E_{a}}{q}$.

Equation (3) takes into account self-heating and below we show that it fully describes the observed saturation and turnover effects. Note that Eq. (3) is valid only at opencircuit conditions and the extracted parameters cannot reproduce the full $J-V$ characteristics with the ideal Shockley equation. However, they can be used in combination with more elaborated models, e.g., for transportlimited solar cells that include charge-carrier mobilities and recombination rates [9] or for drift-diffusion simulations. To fit the measurement data with Eq. (3), it is convenient to reduce the number of fitting parameters by substituting $I_{00}$ in Eq. (3) with Eq. (1), taking a reference point on the experimental curve $\left(V_{\text {ref }}, I_{\text {ref }}\right)$, where self-heating is negligible $(\Delta T<0.5 \mathrm{~K})$.

$$
\begin{aligned}
V_{\mathrm{OC}}\left(I_{\text {Sun }}\right)= & \left(T_{\text {amb }}+I_{\text {Sun }} \Theta_{\mathrm{th}}^{*}\right) \frac{n_{i d} k}{q} \\
& \times\left[\ln \left(\frac{I_{\text {Sun }}}{I_{\text {ref }}}\right)+\frac{q V_{\text {ref }}-E_{a}}{n_{\text {id }} k T_{\text {amb }}}\right]+\frac{E_{a}}{q} .
\end{aligned}
$$

Using Eq. (4), we can describe the experimental data for all investigated solar-cell technologies. Table I summarizes the values of the fitting parameters $E_{a}, n_{\mathrm{id}}$, and $\Theta_{\mathrm{th}}^{*}$. Both representative small-molecule organic solar cells, DCV5T:C $\mathrm{C}_{60}[24,25]$ and F4-ZnPc: $\mathrm{C}_{60}$ [26], show ideality factors close to 1 , comparable to the ones observed previously at solar intensities for a $\mathrm{ZnPc}: \mathrm{C}_{60}$ blend [27]. For both organic solar cells, activation energies nicely
TABLE I. Fitting parameters of the experimental data shown in Fig. 2 according to Eq. (3). $\Theta_{\mathrm{th}, c}^{*}$ and $\Theta_{\mathrm{th}, p}^{*}$ correspond to the effective thermal resistance of the pulsed and continuous Suns- $V_{\mathrm{OC}}$ measurement, respectively.

\begin{tabular}{lcccc}
\hline \hline Material & $n_{\mathrm{id}}$ & $E_{a}(\mathrm{eV})$ & $\Theta_{\mathrm{th}, c}^{*}(\mathrm{~K} /$ Sun $)$ & $\Theta_{\mathrm{th}, p}^{*}(\mathrm{~K} / \mathrm{Sun})$ \\
\hline DCV5T:C 60 & 1.19 & 1.44 & 1.10 & 0.20 \\
F4-ZnPc:C 60 & 1.12 & 1.31 & 1.33 & 0.18 \\
Silicon & 1.34 & 1.21 & 3.58 & 0.49 \\
Perovskite & 1.57 & 1.65 & 2.97 & 0.80 \\
\hline \hline
\end{tabular}

correspond to values obtained from temperature-dependent Suns- $V_{\mathrm{OC}}$ measurements as described by Tvingstedt and Deibel [12] (see Fig. 1 for F4-ZnPc: $\mathrm{C}_{60}$; for DCV5T: $\mathrm{C}_{60}$ see Supplemental Material, part III [20]). However, here we use an adapted technique, which requires no external temperature control and solely knowledge of the ambient temperature. Since both small-molecule devices have the same device architecture and substrate, the effective thermal resistances of both samples are indeed very similar, on the order of $1 \mathrm{~K} / \mathrm{Sun}$. Even below intensities of 10 Suns we see deviations from the isothermal curves, which already originate from a photoinduced sample heating. For the perovskite solar cell [28], we find an ideality factor of 1.57 , a bit lower than reported elsewhere [29]. We determine an activation energy of $1.65 \mathrm{eV}$, which is close to the expected gap for this material system [30]. The effective thermal resistance is among the highest of the investigated devices, which can be explained by the very strong absorption of the perovskite [see Eq. (2)]. The same applies for the silicon photodiode, with an about threefold effective thermal resistance as compared to the organic representatives. We are able to fit the data with a band gap of $1.21 \mathrm{eV}$, which is slightly higher than the well-known band gap for crystalline $\mathrm{Si}$ of $1.12 \mathrm{eV}$ [31]. However, a similar value of $1.23 \mathrm{eV}$ has been determined from temperature-dependent voltage measurements [32]. For a solution-processed solar cell based on P3HT:PCBM, a material system with comparably low absorption, we barely see a voltage turnover, but a saturation of $V_{\mathrm{OC}}$ is present at 30 Suns (see Supplemental Material, part IV [20]). Based on our model, we can estimate temperature changes of up to $100 \mathrm{~K}$ for the highest intensities used in this work (at $\approx 35$ Suns for the perovskite sample). The voltage turnover itself typically happens at smaller temperature rises $\Delta T$ in the range of 20 to $30 \mathrm{~K}$. Overall, we are able to extract a single global ideality factor for this intensity range, despite changes in the slope of the Suns- $V_{\mathrm{OC}}$ measurements.

In order to further verify our model, we perform a second measurement where we minimize self-heating. Instead of keeping the intensity constant at each point for $30 \mathrm{~s}$, we illuminate the devices with a rather short pulse of a few hundred milliseconds, followed by $30 \mathrm{~s}$ of darkness. These pulsed Suns- $V_{\mathrm{OC}}$ measurements are depicted as blue squares in Fig. 2. When pulsing the incident light, none 
of the investigated solar cells heats up sufficiently to show a voltage turnover. This indicates that neither the contact of the solar cell nor approaching the built-in voltage can deliver a full explanation of the voltage turnover. Still, our model is able to describe these pulsed Suns- $V_{\mathrm{OC}}$ measurements with a lower effective thermal resistance (Table I). It is also evident, that even in the pulsed measurement the data points deviate from the ideal isothermal case (gray solid line). Our automated measurement routine produces light pulses with a length of about $500 \mathrm{~ms}$. In order to prove whether there still is a significant self-heating effect leading to deviations from the isothermal curve, we perform timeresolved measurements of the open-circuit voltage within the $35 \mathrm{~s}$ upon illumination of the $\mathrm{F} 4-\mathrm{ZnPc}: \mathrm{C}_{60}$ and perovskite solar-cell sample for different fixed intensities (Fig. 3). For F4-ZnPc:C 60 [Fig. 3(a)] we find voltage drops of a few millivolts already after a few $100 \mathrm{~ms}$, a change sufficiently high to affect the pulsed (blue) measurement in Fig. 2. After $500 \mathrm{~ms}$, the solar cells already lose a few millivolts as compared to the maximum values at high intensities. In contrast to $\mathrm{F} 4-\mathrm{ZnPc}: \mathrm{C}_{60}$, perovskite-based solar cells need much longer to reach an electronic steady state [33]. Any measurement with shortly applied pulses would characterize these cells in an electronic nonequilibrium (see Supplemental Material, part VII [20]), making a comparison between different structures and material compositions impossible. For this reason, the pulsed measurement is done with a 2-s pulse, which is sufficient to reach the electronic steady state. However, it makes the device more vulnerable to self-heating and thus explains the higher effective thermal resistance (Table I). This finding highlights the necessity of our model for material systems

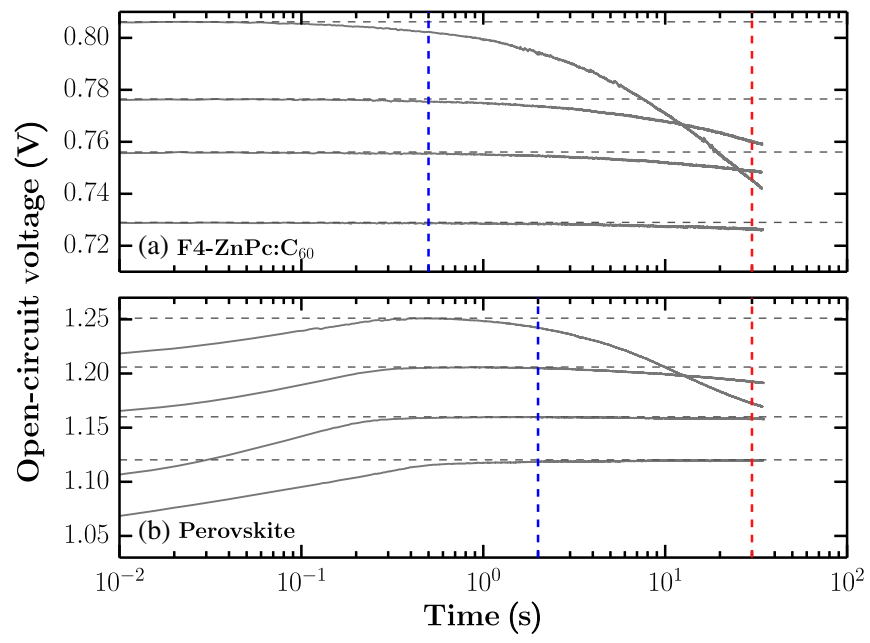

FIG. 3. Dependence of the open-circuit voltage as a function of time for various intensities for (a) the $\mathrm{F} 4-\mathrm{ZnPc}: \mathrm{C}_{60}$ sample and (b) for the perovskite sample. Because of self-heating and a consequential increase of the cell temperature, $V_{\mathrm{OC}}$ decreases over time. The blue and red dashed lines mark the point in time at which $V_{\mathrm{OC}}$ is measured in the pulsed and continuous Suns- $V_{\mathrm{OC}}$ measurement, respectively. where long light pulses are needed to reach the electronic steady state. With our approach, one can directly benefit from a temperature change, thus, self-heating is not to be prevented. Depending on the measurement conditions, such as the light intensity and geometry and heat dissipation of the sample, it can be necessary to use time-resolved measurement techniques to investigate the solar cell close to isothermal conditions.

In order to understand the physical origin of the voltage turnover at high light intensities in more detail, we show in Fig. 4 a plot of Eq. (3) for different effective thermal resistances. In the absence of absorption-induced selfheating, i.e., $\Theta_{\mathrm{th}}^{*}=0$, the open-circuit voltage scales linearly with the logarithm of the illumination intensity as illustrated by dotted black lines in Fig. 4. Upon changing the ambient temperature $T_{\mathrm{amb}}$, the slope will change but all curves will ultimately cross the $\left(E_{a}, I_{00}\right)$. With increasing effective thermal resistance, the solar cell will heat up, reducing its open-circuit voltage, and eventually leading to a voltage turnover. Such an electrothermal feedback mechanism can also be found in thermistor devices as well as in organic light-emitting diodes, where the power dissipation increases faster than the heat can be conducted [34,35].

Taking the derivative of Eq. (3) gives an expression for the intensities of the turnover points $\mathrm{TOP}_{1}$ and $\mathrm{TOP}_{2}$ :

$$
\mathrm{TOP}_{1,2}=I_{00}\left[W_{0,-1}\left(-\frac{e T}{\Theta_{\mathrm{th}}^{*} I_{00}}\right)-1\right],
$$

where $W_{0}$ and $W_{-1}$ are the upper and lower branch of the Lambert $W$ function [36], respectively, and $e$ is Euler's number. For $W_{0,-1}=-1$, both turnover points coincide,

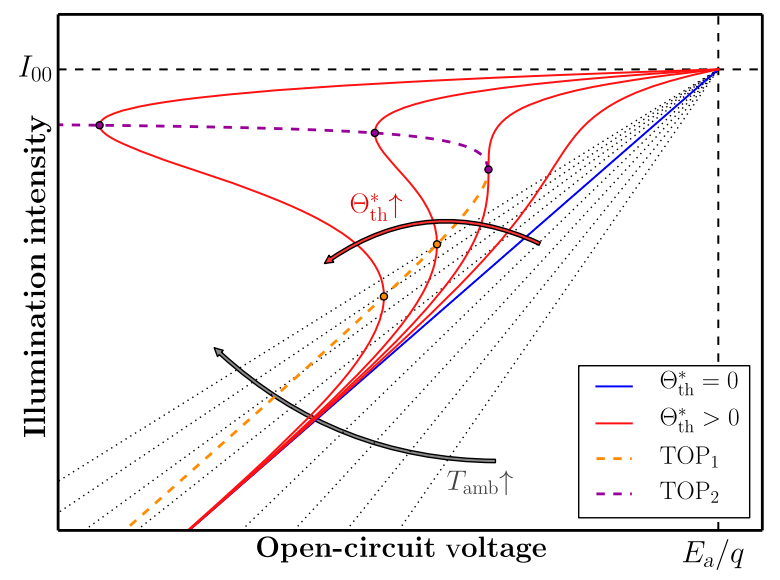

FIG. 4. Schematic illustration of the self-heating model. The black dotted lines show the isothermal behavior for different temperatures. All curves meet in the point $\left(I_{00}, E_{a} / q\right)$. Adding absorption-induced self-heating results in deviations from the isothermal curves (red lines), i.e., the voltage decreases with increasing self-heating coefficient $\Theta_{\text {th }}^{*}$. The turnover points $\mathrm{TOP}_{1,2}$ (orange and purple dashed line) exists for $\Theta_{\mathrm{th}}^{*}>e^{2} T_{\mathrm{amb}} /$ $I_{00}$; their dependence on $\Theta_{\mathrm{th}}^{*}$ and $T_{\mathrm{amb}}$ are shown in the Supplemental Material, part V [20]. 
which is the case when $\Theta_{\mathrm{th}, 0}^{*}=e^{2} T / I_{00}$ (see Supplemental Material [20] for more details), while no turnover points are defined for thermal resistances smaller than $\Theta_{\mathrm{th}, 0}^{*}$. The intensity at which the first turnover point $\left(\mathrm{TOP}_{1}\right)$ occurs, decreases for increasing effective thermal resistance and lower ambient temperatures (see Supplemental Material [20] for more details). Therefore, the effect is even stronger when conducting Suns- $V_{\text {OC }}$ measurements at low ambient temperatures, i.e., the turnover appears at lower light intensities. Indeed, we find data in the literature which support this trend [12-14]. The second turnover point $\left(\mathrm{TOP}_{2}\right)$ will always appear very close to $I_{00}$, thus making it very unlikely to reach this point experimentally (see Supplemental Material [20]).

In conclusion, we present a model that includes absorption-induced self-heating for the analysis of Suns- $V_{\mathrm{OC}}$ measurement. It explains the saturation of the open-circuit voltage with increasing light intensities for various photovoltaic technologies. The results reveal that commonly invoked causes such as surface recombination, the density of states, or the back contact are not necessarily needed. While it is common to control the temperature of solar cells to determine the performance according to standard testing conditions, we want to emphasize the importance to also consider self-heating in other measurement techniques. In particular, we show that for the perovskite device, longer illumination durations are required to reach a steady state which inevitably leads to self-heating. Additionally, our adapted Suns- $V_{\mathrm{OC}}$ measurements also allow us to determine the effective gap from a single curve at ambient temperature, which is of special interest for alternative technologies such as small-molecule organic, polymer, and perovskite solar cells.

This work received funding from the German Federal Ministry for Education and Research (BMBF) through the InnoProfile projekt "Organische p-i-n Bauelemente 2.2," the German Research Foundation (DFG) within the Cluster of Excellence Center for Advancing Electronics Dresden (cfaed), and the DFG project EFOD (RE 3198/6-1). Furthermore, we acknowledge financial support from the European Union H2020 project INFORM (Grant No. 675867), the Spanish Ministry of Economy and Competitiveness (MINECO) via the Unidad de Excelencia Mara de Maeztu MDM-2015-0538, MAT2017-88821-R and PCIN-2015-255, and the Generalitat Valenciana (Prometeo/2016/135). J. A. thanks the Spanish Ministry of Education, Culture and Sport for support from a predoctoral grant.

[1] William Shockley and Hans J. Queisser, Detailed balance limit of efficiency of $p$ - $n$ junction solar cells, J. Appl. Phys. 32, 510 (1961).
[2] Martin A. Green, Radiative efficiency of state-of-the-art photovoltaic cells, Prog. Photovoltaics 20, 472 (2012).

[3] David L. King, Jay A. Kratochvil, and William E. Boyson, in Proceedings of the IEEE 26th Photovoltaic Specialists Conference, Anaheim, CA, 1997 (IEEE, Piscataway, NJ, 1997), pp. 1183-1186.

[4] Erdem Cuce, Pinar Mert Cuce, and Tulin Bali, An experimental analysis of illumination intensity and temperature dependency of photovoltaic cell parameters, Appl. Energy 111, 374 (2013).

[5] Xingshu Sun, Yubo Sun, Zhiguang Zhou, Muhammad Ashraful Alam, and Peter Bermel, Radiative sky cooling: Fundamental physics, materials, structures, and applications, Nanophotonics 6, 997 (2017).

[6] E. A. Katz, D. Faiman, S. M. Tuladhar, J. M. Kroon, M. M. Wienk, T. Fromherz, F. Padinger, C. J. Brabec, and N. S. Sariciftci, Temperature dependence for the photovoltaic device parameters of polymer-fullerene solar cells under operating conditions, J. Appl. Phys. 90, 5343 (2001).

[7] I. Riedel, J. Parisi, V. Dyakonov, L. Lutsen, D. Vanderzande, and J.C.C. Hummelen, Effect of temperature and illumination on the electrical characteristics of polymer-fullerene bulk-heterojunction solar cells, Adv. Funct. Mater. 14, 38 (2004).

[8] R. A. Sinton and A. Cuevas, A quasi-steady-state opencircuit voltage method for solar cell characterization, in Proceedings of the 16th European Photovoltaic Solar Energy Conference, Glasgow, UK, 2000 (Earthscan Publications Ltd., London, 2001), pp. 1152-1155.

[9] Uli Würfel, Dieter Neher, Annika Spies, and Steve Albrecht, Impact of charge transport on current-voltage characteristics and power-conversion efficiency of organic solar cells, Nat. Commun. 6, 6951 (2015).

[10] Sebastian Schiefer, Birger Zimmermann, and Stefan W Glunz, Applicability of the Suns- $V_{\text {oc }}$ method on organic solar cells, IEEE J. Photovoltaics 4, 271 (2014).

[11] Sebastian Schiefer, Birger Zimmermann, and Uli Würfel, Determination of the intrinsic and the injection-dependent charge carrier density in organic solar cells using the Suns- $V_{\text {oc }}$ method, J. Appl. Phys. 115, 044506 (2014).

[12] Kristofer Tvingstedt and Carsten Deibel, Temperature dependence of ideality factors in organic solar cells and the relation to radiative efficiency, Adv. Energy Mater. 6, 1502230 (2016).

[13] S. W. Glunz, J. Nekarda, H. Mäckel, and A. Cuevas, Analyzing back contacts of silicon solar cells by Suns- $V_{\text {oc }}$-measurements at high illumination densities, in Proceedings of the 22nd European Photovoltaic Solar Energy Conference, Milan, Italy, 2007 (WIP-Renewable Energies, München, 2007), pp. 849-853.

[14] Oki Gunawan, Tayfun Gokmen, and David B. Mitzi, Suns- $V_{\text {oc }}$ characteristics of high performance kesterite solar cells, J. Appl. Phys. 116, 084504 (2014).

[15] A. Wagenpfahl, D. Rauh, M. Binder, C. Deibel, and V. Dyakonov, S-shaped current-voltage characteristics of organic solar devices, Phys. Rev. B 82, 115306 (2010).

[16] Alexander Wagenpfahl, Carsten Deibel, and Vladimir Dyakonov, Organic solar cell efficiencies under the aspect of reduced surface recombination velocities, IEEE J. Sel. Top. Quantum Electron. 16, 1759 (2010). 
[17] T. Kirchartz and J. Nelson, Meaning of reaction orders in polymer: Fullerene solar cells, Phys. Rev. B 86, 165201 (2012).

[18] Xingshu Sun, Timothy J. Silverman, Zhiguang Zhou, Mohammad Ryyan Khan, Peter Bermel, and Muhammad Ashraful Alam, An optics-based approach to thermal management of photovoltaics: Selective-spectral and radiative cooling, IEEE J. Photovoltaics 7, 566 (2017).

[19] Raghu Vamsi Krishna Chavali, Joan V. Li, Corsin Battaglia, Stefaan De Wolf, Jeffrey Lynn Gray, and Muhammed Ashraful Alam, A generalized theory explains the anomalous Suns- $V_{\text {oc }}$ response of $\mathrm{Si}$ heterojunction solar cells, IEEE J. Photovoltaics 7, 169 (2017).

[20] See Supplemental Material at http://link.aps.org/ supplemental/10.1103/PhysRevApplied.9.051003 for further information about the investigated solar cells, the derivation of the fitting model, and further temperature dependent measurements.

[21] Axel Fischer, Paul Pahner, Björn Lüssem, Karl Leo, Reinhard Scholz, Thomas Koprucki, Jürgen Fuhrmann, Klaus Gärtner, and Annegret Glitzky, Self-heating effects in organic semiconductor crossbar structures with small active area, Org. Electron. 13, 2461 (2012).

[22] F. F. Oettinger and D. L. Blackburn, Thermal Resistance Measurements, NIST Special Publications Vol. 400-86 (NIST, Gaithersburg, MD, 1990).

[23] European Committee for Electrotechnical Standardization, IEC 60904-3:2016: Measurement Principles for Terrestrial Photovoltaic (PV) Solar Devices with Reference Spectral Irradiance Sata (CEN-CENELEC Management Centre, Brussels, 2016), https://webstore.iec.ch/publication/24526.

[24] Roland Fitzner, Elena Mena-Osteritz, Amaresh Mishra, Gisela Schulz, Egon Reinold, Matthias Weil, Christian Körner, Hannah Ziehlke, Chris Elschner, Karl Leo, Moritz Riede, Martin Pfeiffer, Christian Uhrich, and Peter Bäuerle, Correlation of $\pi$-conjugated oligomer structure with film morphology and organic solar cell performance, J. Am. Chem. Soc. 134, 11064 (2012).

[25] Rico Meerheim, Christian Körner, and Karl Leo, Highly efficient organic multi-junction solar cells with a thiophene based donor material, Appl. Phys. Lett. 105, 063306 (2014).

[26] Jan Meiss, Andre Merten, Moritz Hein, Christoph Schuenemann, Stefan Schäfer, Max Tietze, Christian Uhrich, Martin Pfeiffer, Karl Leo, and Moritz Riede, Fluorinated zinc phthalocyanine as donor for efficient vacuum-deposited organic solar cells, Adv. Funct. Mater. 22, 405 (2012).

[27] Wolfgang Tress, Karl Leo, and Moritz Riede, Dominating recombination mechanisms in organic solar cells based on ZnPc and C60, Appl. Phys. Lett. 102, 163901 (2013).

[28] Cristina Momblona, Lidón Gil-Escrig, Enrico Bandiello, Eline M. Hutter, Michele Sessolo, Kay Lederer, Jan Blochwitz-Nimoth, and Henk J. Bolink, Efficient vacuum deposited $p-i-n$ and $n-i-p$ perovskite solar cells employing doped charge transport layers, Energy Environ. Sci. 9, 3456 (2016).

[29] Sumanshu Agarwal, Madhu Seetharaman, Naresh K. Kumawat, Anand S. Subbiah, Shaibal K. Sarkar, Dinesh Kabra, Manoj A. G. Namboothiry, and Pradeep R. Nair, On the uniqueness of ideality factor and voltage exponent of perovskite-based solar cells, J. Phys. Chem. Lett. 5, 4115 (2014).

[30] Kristofer Tvingstedt, Olga Malinkiewicz, Andreas Baumann, Carsten Deibel, Henry J. Snaith, Vladimir Dyakonov, and Henk J. Bolink, Radiative efficiency of lead iodide based perovskite solar cells, Nat. Sci. Rep. Ochanomizu Univ. 4, 1 (2014).

[31] Y.P. Varshni, Temperature dependence of the energy gap in semiconductors, Physica (Amsterdam) 34, 149 (1967).

[32] Jürgen W. Precker and Marclio A. da Silva, Experimental estimation of the band gap in silicon and germanium from the temperature-voltage curve of diode thermometers, Am. J. Phys. 70, 1150 (2002).

[33] Wolfgang Tress, Metal halide perovskites as mixed electronic-ionic conductors: Challenges and opportunitiesFrom hysteresis to memristivity, J. Phys. Chem. Lett. 8, 3106 (2017).

[34] A. Fischer, P. Pahner, B. Lüssem, K. Leo, R. Scholz, T. Koprucki, K. Gärtner, and A. Glitzky, Self-Heating, Bistability, and Thermal Switching in Organic Semiconductors, Phys. Rev. Lett. 110, 126601 (2013).

[35] Axel Fischer, Thomas Koprucki, Klaus Gärtner, Max L. Tietze, Jacqueline Brückner, Björn Lüssem, Karl Leo, Annegret Glitzky, and Reinhard Scholz, Feel the heat: Nonlinear electrothermal feedback in organic LEDs, Adv. Funct. Mater. 24, 3367 (2014).

[36] R. M. Corless, G. H. Gonnet, D. E. G. Hare, D. J. Jeffrey, and D. E. Knuth, On the Lambert W function, Adv. Comput. Math. 5, 329 (1996). 